\section{Historia del Hospital San Francisco de Borja de Santiago de Chile (1772-1944)}

\author{
Laval Manrique, Enrique \\ Ediciones Universidad Católica de Chile. 2011.
}

El doctor Enrique Laval Manrique fue el fundador del Museo de la Medicina del Servicio Nacional de Salud y de la Sociedad Chilena de Historia de la Medicina, en 1955. Creador, alma y realizador de los Anales Chilenos de Historia de la Medicina, cuyo primer número apareció en 1959. En 1988, las colecciones del Museo y la biblioteca pasaron a formar parte del Museo Nacional de Historia de la Medicina, ubicado en la Facultad de Medicina de la Universidad de Chile y que lleva su nombre.

En el conjunto de escritos inéditos del doctor Enrique Laval Manrique (1895-1970) se encontraba el trabajo acerca de la Historia del Hospital San Francisco de Borja de Santiago, que junto con la del Hospital San Juan de Dios, publicada en 1949, completan el acontecer y devenir de los dos establecimientos hospitalarios más antiguos de Chile.

Es necesario destacar el conocimiento de primera mano que tenía el doctor Laval Manrique de muchos hospitales a través de documentos administrativos que pasaban por su oficina, en las numerosas visitas de inspección y distintas funciones ejercidas durante toda su época de labor en la antigua Beneficencia, donde llegó a ser Jefe del Departamento Médico. Todo esto lo impulsó a redactar los trabajos generados en la búsqueda permanente en archivos, bibliotecas y bodegas institucionales.

El libro comienza con el relato de la iniciativa de trasladar la sección mujeres del Hospital San Juan de Dios a uno destinado exclusivamente a ellas, inaugurándose el nuevo establecimiento en 1782, en el edificio del Noviciado de San Francisco de Borja, que se encontraba vacante como consecuencia de la expulsión de los miembros de la Compañía de Jesús y situado en la Cañada (hoy Avenida del Libertador Bernardo O’Higgins) al poniente de la calle San Ignacio.

A lo largo de los capítulos aparece la evolución de la actividad del hospital (composición del personal, movimientos de pacientes, listas de enfermedades que los aquejaban, bienes, insumos y presupuestos). El autor presenta la semblanza de administradores y facultativos que impulsaron el crecimiento y avances técnicos, sin eludir los numerosos problemas como la demanda de camas durante las epidemias y a consecuencia de los heridos en los conflictos armados.

En el capítulo III, se da cuenta de la construcción y del traslado del hospital, en 1859, a un nuevo emplazamiento en la Cañada, a unos dos kilómetros al oriente de su ubicación anterior. En el capítulo IV se analiza con detalle la situación obstétrica en Santiago, el súbito traslado de las parturientas, en 1873, desde un edificio del barrio Yungay a una sala del Hospital San Borja y luego la construcción, en 1875, de lo que se conocería como la Maternidad del Hospital San Francisco de Borja.

El capítulo V refiere la vida hospitalaria en las postrimerías del siglo XIX y los primeros decenios del siglo XX, mencionando su transformación en un centro asistencial mixto mediante la habilitación de sectores para hombres.

A pesar de que el relato se detiene en 1944, sin contener párrafos de cierre, sugiriendo que el autor no consideraba terminada su obra, se estimó que apreciando la importancia de la numerosa información aportada, se justificaba plenamente ponerla a disposición de los lectores interesados.

El Programa de Estudios Médicos Humanísticos de la Facultad de Medicina de la Pontificia Universidad Católica de Chile, decidió publicar esta obra, que constituye una valiosa fuente para la historia de las instituciones hospitalarias nacionales.

Ignacio Duarte García de Cortázar Programa de Estudios Médicos Humanísticos Universidad Católica de Chile 